\title{
Quasi-Free-Standing Graphene Monolayer on a Ni Crystal through Spontaneous Na Intercalation
}

\author{
Young S. Park, ${ }^{1}$ Jae H. Park,${ }^{2}$ Han N. Hwang, ${ }^{3}$ Tomba Singh Laishram, ${ }^{4}$ Kwang S. Kim,,${ }^{4, \dagger}$ \\ Myung H. Kang, ${ }^{2}$ and Chan C. Hwang ${ }^{3, *}$ \\ ${ }^{1}$ School of Life Sciences, Ulsan National Institute of Science and Technology, Ulsan 689-798, Korea \\ ${ }^{2}$ Department of Physics, Pohang University of Science and Technology, Pohang 790-784, Korea \\ ${ }^{3}$ Beamline Research Division, Pohang Accelerator Laboratory, \\ Pohang University of Science and Technology, Pohang 790-784, Korea \\ ${ }^{4}$ Center for Superfunctional Materials, Department of Chemistry and Department of Physics, \\ School of Natural Science, Ulsan National Institute of Science and Technology (UNIST), \\ Ulsan 689-798, Korea
}

(Received 24 August 2013; revised manuscript received 25 May 2014; published 29 July 2014)

\begin{abstract}
Graphene on metal substrates often shows different electronic properties from isolated graphene because of graphene-substrate interactions. One needs to remove the metals with acids and then to transfer graphene to weakly interacting substrates to recover electrical properties inherent in graphene. This process is not easy and besides causes undesirable tears, defects, and impurities in graphene. Here, we report a method to recover the electronic structure of graphene from a strongly interacting $\mathrm{Ni}$ substrate by spontaneous $\mathrm{Na}$ intercalation. In order to characterize the intercalation process, the density-functional-theory calculations and angle-resolved photoemission-spectroscopy (ARPES) and scanning-tunneling-microscopy (STM) measurements are carried out. From the density-functional-theory calculations, Na atoms energetically prefer interface intercalation to surface adsorption for the graphene/Ni(111) surface. Unlike most intercalants, $\mathrm{Na}$ atoms intercalate spontaneously at room temperature due to a tiny diffusion barrier, which is consistent with our temperature-dependent ARPES and core-level photoemission spectroscopy, and with our submonolayer ARPES and STM results at room temperature. As a result of the spontaneous intercalation, the electronic structure of graphene is almost recovered, as confirmed by the Dirac cone with a negligible band gap in ARPES and the sixfold symmetry in STM.
\end{abstract}

DOI: 10.1103/PhysRevX.4.031016

\section{INTRODUCTION}

The discovery of unique transport properties of graphene prepared by mechanical exfoliation has spurred many new research activities for future electronic devices because of graphene's intriguing energy band structure showing high carrier mobility [1]. Many efforts have been devoted to growing high-quality and large-area graphene on $\mathrm{SiC}$ [2-4] and metal substrates [5-10]. To date, chemical vapor deposition (CVD) on metallic substrates has been the most efficient method to produce large-area graphene layers [6-8]. $\mathrm{Ni}$ and $\mathrm{Cu}$ surfaces among metal substrates are the most representative examples for large-scale graphene synthesis because of the transfer ability to other substrates $[11,12]$. However, the quality of graphene grown on foils or

\footnotetext{
*chwang@postch.ac.kr

${ }^{\dagger}$ kimks@unist.ac.kr
}

Published by the American Physical Society under the terms of the Creative Commons Attribution 3.0 License. Further distribution of this work must maintain attribution to the author(s) and the published article's title, journal citation, and DOI.
Subject Areas: Computational Physics,

Condensed Matter Physics, Graphene films of these substrates by CVD is not as high as that of graphene obtained by mechanical exfoliation, due to the existence of domains with different crystallographic orientations. A recent report on graphene grown by CVD showed lots of grain boundaries consisting of StoneWhales-type defects [13]. In particular, graphene grown on even $\mathrm{Cu}$ single crystals has multidomain structures with different orientations due to weak interactions between carbon atoms in graphene and $\mathrm{Cu}$ atoms [14].

On the other hand, since the lattice of graphene is well matched to that of $\mathrm{Ni}$, graphene grown on a single-crystal $\mathrm{Ni}$ (111) surface does not show different in-plane orientations [15]. However, the unique electronic structure of graphene exhibiting linear dispersion disappears due to the strong interaction of graphene with the $\mathrm{Ni}$ substrate [16]. To restore the pristine electronic structure of graphene, it is essential to remove the substrate with acids and then to transfer graphene to weakly interacting substrates. However, removing the metal substrate from a graphene/metal system and transferring the graphene to other substrates is not an easy task [6,11], and these processes can cause undesirable tears, defects, and impurities in graphene $[17,18]$. 
Some metals have been reported to be intercalated between graphene and a Ni substrate. For example, noble metals such as $\mathrm{Au}, \mathrm{Ag}$, and $\mathrm{Cu}$ were experimentally and theoretically found to be intercalated [19-25]. In particular, $\mathrm{Au}$ intercalation was observed to recover the Dirac cone close to that of the freestanding graphene near the Fermi energy [22-24]. Al also gives rise to a similar Dirac-cone structure after intercalation [26]. In the case of alkali metals, energy gaps of approximately $1.3,0.8$, and 0.7 $\mathrm{eV}$ were observed for $\mathrm{Na}, \mathrm{K}$, and $\mathrm{Cs}[27,28]$, respectively.

In this paper, we show that $\mathrm{Na}$ can be used to restore the electronic structure of graphene from a strongly interacting $\mathrm{Ni}(111)$ surface that is characterized by using densityfunctional-theory (DFT) calculations, angle-resolved photoemission spectroscopy (ARPES), core-level photoemission spectroscopy (PES), and scanning tunneling microscopy (STM). From the DFT calculations, Na atoms energetically prefer interface intercalation to surface adsorption. When compared to noble metals and $\mathrm{Al}$, which require thermal annealing at elevated temperatures of a few hundreds of ${ }^{\circ} \mathrm{C}$ for intercalation, $\mathrm{Na}$ atoms adsorbed on the graphene surface penetrate into the graphene far below room temperature (approximately $-100^{\circ} \mathrm{C}$ ), and thus intercalation takes place spontaneously even at room temperature. This seems to be closely related with a tiny diffusion barrier for $\mathrm{Na}$ atoms. The spontaneous $\mathrm{Na}$ intercalation makes the graphene layer nearly electronically ideal, thereby showing its inherent sixfold symmetry and characteristic bands featuring the Dirac-cone structure with a negligible band gap. Because the $\mathrm{Na}$ layer can be easily oxidized even in ultrahigh vacuum, one can realize an ideal graphene on an insulating support without etching the $\mathrm{Ni}$ substrate and then transfer the graphene to other insulating substrates.

\section{DFT CALCULATIONS}

\section{A. Computational scheme}

We carry out DFT calculations by employing the Vienna $a b$ initio simulation package [29] with the use of the spinpolarized local density approximation and the projectoraugmented-wave method with a plane-wave basis set of $400 \mathrm{eV}$ [30,31]. The $\mathrm{Ni}(111)$ surface is simulated by a periodic slab geometry with a slab thickness of five atomic layers and a vacuum spacing of about $16 \AA$. The $k$-point sampling is done on a $4 \times 4 \times 1$ mesh for the $\mathrm{Ni}(111) 2 \times 2$ supercell. All atoms but the bottom two Ni layers are relaxed until the residual force components are within $0.03 \mathrm{eV} / \AA$. The lattice mismatch between freestanding graphene (2.45 $\AA$ ) and $\mathrm{Ni}(111)(2.42 \AA)$ is $1.2 \%$ [22]. The graphene is set to match the lattice of the $\mathrm{Ni}(111)$ surface. The reversematching procedure is found to produce no noticeable difference in the calculated graphene $\pi$ band of the graphene/Na/Ni(111) surface.

\section{B. Structural model of the graphene/Ni(111) substrate}

For the graphene/ $\mathrm{Ni}(111)$ surface, the $\mathrm{C}$ atoms of graphene are aligned on the top and fcc sites of $\mathrm{Ni}(111)$. This top-fcc model was proposed in a low-energy electrondiffraction study by Gamo et al. [32] and has been supported by several DFT studies as the most stable adsorption configuration [33-35]. Our calculated adsorption energy of $0.09 \mathrm{eV} / \mathrm{C}$ and graphene-Ni interlayer spacing of $2.08 \AA$ are in good agreement with previous DFT studies [33-35].

\section{Optimized structures and energetics}

$\mathrm{Na}$ atoms doped on graphene/Ni(111) may adsorb on the graphene layer or intercalate between the graphene and the Ni(111) substrate. Therefore, in the present calculations, we have considered both (adsorption and intercalation) possibilities for given $\mathrm{Na}$ coverages. Figure 1 shows the intercalated graphene $/ \mathrm{Na} / \mathrm{Ni}(111)$ structures obtained for different coverages. As seen in Figs. 1(a)-(d), the intercalated $\mathrm{Na}$ atoms prefer the hcp hollow site of $\mathrm{Ni}(111)$ just below the center of the graphene hexagon. The case of 1.07 ML [Fig. 1(e)] is rather exceptional. Since the energy difference between the top and hollow sites for an isolated $\mathrm{Na}$ atom is as small as $0.06 \mathrm{eV}$, a hexagonal arrangement of
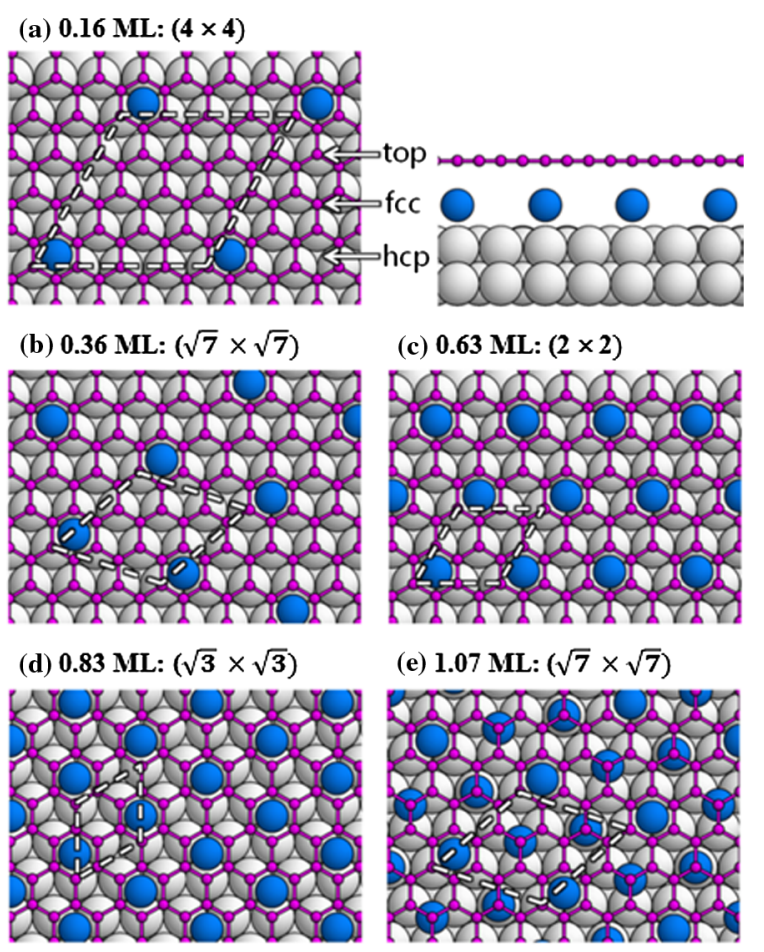

(e) $1.07 \mathrm{ML}:(\sqrt{7} \times \sqrt{7})$

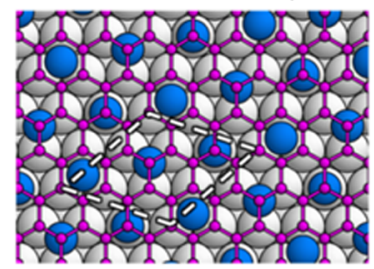

FIG. 1. Employed structural models for the Na-intercalated graphene/Ni(111) surface. Large, medium, and small balls represent the $\mathrm{Ni}, \mathrm{Na}$, and $\mathrm{C}$ atoms, respectively. $1 \mathrm{ML}$ denotes one $\mathrm{Na}$ atom per five $\mathrm{C}$ atoms. Dashed lines represent the corresponding unit cells. Arrows in (a) indicate three highsymmetry surface sites. 
$\mathrm{Na}$ is energetically preferred due to the $\mathrm{Na}-\mathrm{Na}$ repulsion in such a dense coverage. For the adsorption cases, $\mathrm{Na}$ atoms prefer the center of the graphene hexagon from 0.16 to $0.83 \mathrm{ML}$, and a hexagonal arrangement of $\mathrm{Na}$ is energetically preferred at $1.07 \mathrm{ML}$. The resulting energy-optimized $\mathrm{Na}$ configurations have the same lateral positions as the structures shown in Fig. 1, with the $\mathrm{Na}$ atoms placed over the graphene/Ni(111) surface.

Table I shows the interlayer spacing of the Na/graphene/ $\mathrm{Ni}(111)$ and graphene/ $\mathrm{Na} / \mathrm{Ni}(111)$ surfaces. When adsorbed on the graphene surface, the equilibrium Nagraphene distance increases from $2.26 \AA$ at $0.16 \mathrm{ML}$ to $2.64 \AA$ at $1.07 \mathrm{ML}$, indicating that the bonding between $\mathrm{Na}$ and graphene weakens with the coverage due to stronger $\mathrm{Na}-\mathrm{Na}$ bond formations. It is notable that the adsorbed $\mathrm{Na}$ atoms little affect the structure of the graphene/Ni(111) substrate. $\mathrm{Na}$ intercalations lead to a large separation of graphene from the Ni substrate: The resulting graphene-Na and graphene-Ni distances are about 2.4 and $4.8 \AA$, respectively, which are somewhat insensitive to the $\mathrm{Na}$ coverage. With the relatively large graphene-Na distance (approximately $2.4 \AA$ ), the interaction of graphene with the $\mathrm{Na} / \mathrm{Ni}(111)$ substrate is likely weaker than with the $\mathrm{Ni}(111)$ substrate.

Figure 2 compares the adsorption and intercalation energies as a function of the Na coverage, which represents the energy gain per $\mathrm{Na}$ atom obtained by adsorption or intercalation $E=-\left(E_{\text {doped }}-E_{\text {clean }}-N \times E_{\mathrm{Na}}\right) / N$, where $E_{\text {doped }}, E_{\text {clean }}$, and $E_{\mathrm{Na}}$ are the total energies of the $\mathrm{Na}$-doped surface, the clean graphene/Ni(111) surface, and a free $\mathrm{Na}$ atom, respectively, and $N$ is the number of $\mathrm{Na}$ atoms in the unit cell. Interestingly, $\mathrm{Na}$ atoms energetically prefer interface intercalation to surface adsorption. Except for the low coverage of $0.16 \mathrm{ML}, \mathrm{Na}$ intercalation is favored over surface adsorption with more energy gains of about $1 \mathrm{eV}$. Even at the coverage of $0.16 \mathrm{ML}$, the adsorption energy $(0.24 \mathrm{eV})$ is much smaller

TABLE I. Structural details of the graphene/Ni(111), Na/ graphene/Ni(111), and graphene/Na/Ni(111) surfaces. $d(\AA)$ represents the interlayer spacing between two species. Here, the layer spacing is averaged over all atoms in the layer.

\begin{tabular}{lccc}
\hline \hline & Coverage & $d(\mathrm{Na}-\mathrm{Gr})$ & $d(\mathrm{Gr}-\mathrm{Ni})$ \\
\hline $\mathrm{Gr} / \mathrm{Ni}(111)$ & $0 \mathrm{ML}$ & & 2.08 \\
$\mathrm{Na} / \mathrm{Gr} / \mathrm{Ni}(111)$ & $0.16 \mathrm{ML}$ & 2.26 & 2.10 \\
& $0.36 \mathrm{ML}$ & 2.30 & 2.11 \\
& $0.63 \mathrm{ML}$ & 2.37 & 2.12 \\
& $0.83 \mathrm{ML}$ & 2.44 & 2.12 \\
& $1.07 \mathrm{ML}$ & 2.64 & 2.12 \\
$\mathrm{Gr} / \mathrm{Na} / \mathrm{Ni}(111)$ & $0.16 \mathrm{ML}$ & 2.42 & 4.84 \\
& $0.36 \mathrm{ML}$ & 2.36 & 4.75 \\
& $0.63 \mathrm{ML}$ & 2.39 & 4.74 \\
& $0.83 \mathrm{ML}$ & 2.43 & 4.83 \\
& $1.07 \mathrm{ML}$ & 2.47 & 4.82 \\
\hline \hline
\end{tabular}

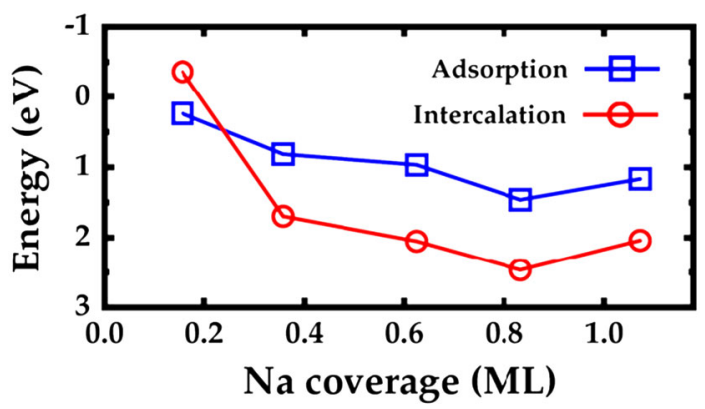

FIG. 2. Adsorption and intercalation energies as a function of the $\mathrm{Na}$ coverage.

than the bulk cohesive energy of $\mathrm{Na}(1.27 \mathrm{eV})$, so $\mathrm{Na}$ atoms may form dense islands rather than forming isolated adsorption states through feasible surface diffusions with such a low diffusion barrier as $0.03 \mathrm{eV}$.

It would be interesting to compare the $\mathrm{Na}$ case with the adsorption and intercalation behavior of $\mathrm{H}$ atoms on the graphene/Ni(111) surface, since $\mathrm{H}$ atoms are also widely used for manipulating the band structure of substratesupported graphenes [36-39]. In our calculations for the adsorption and intercalation of a single $\mathrm{H}$ atom on the graphene/Ni(111)- $(2 \times 2)$ surface, we find that its adsorption energy is $2.75 \mathrm{eV}$, which is energetically comparable with the intercalation energy $(2.83 \mathrm{eV})$. Moreover, the adsorbed $\mathrm{H}$ atom has a large barrier of $0.71 \mathrm{eV}$ for surface diffusion on the graphene layer, which is likely to make the intercalation of $\mathrm{H}$ atoms more difficult than the $\mathrm{Na}$ case. It is worth mentioning the recent ARPES studies for the intercalation of $\mathrm{H}$ and $\mathrm{Na}$ on the graphene/SiC $(0001)$ surface, where $\mathrm{H}$ atoms intercalate at a much higher temperature of $400{ }^{\circ} \mathrm{C}$ [38] while $\mathrm{Na}$ atoms intercalate at $75^{\circ} \mathrm{C}[39]$.

\section{Dirac-point shift and band structures}

Figure 3 shows the Dirac-point shift of graphene/Na/ $\mathrm{Ni}(111)$ as a function of the $\mathrm{Na}$ coverage. In all $\mathrm{Na}$ coverages considered, we find that the graphene $\pi$ bands form a well-defined Dirac point. It is notable that a small intercalation of $0.16 \mathrm{ML}$ is enough to recover the

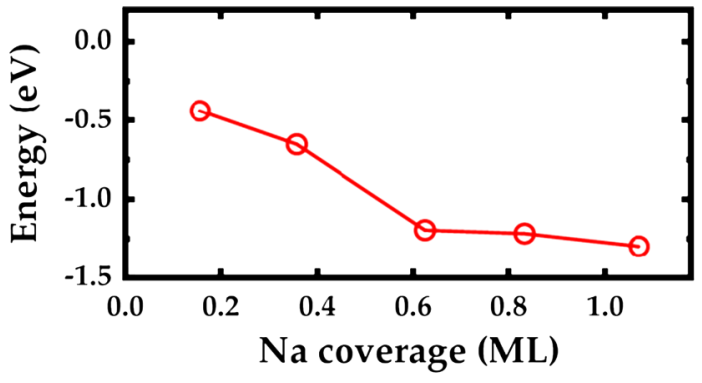

FIG. 3. Dirac-point shift with reference to the Fermi level as a function of the $\mathrm{Na}$ coverage. All data are obtained for the majority-spin band. 


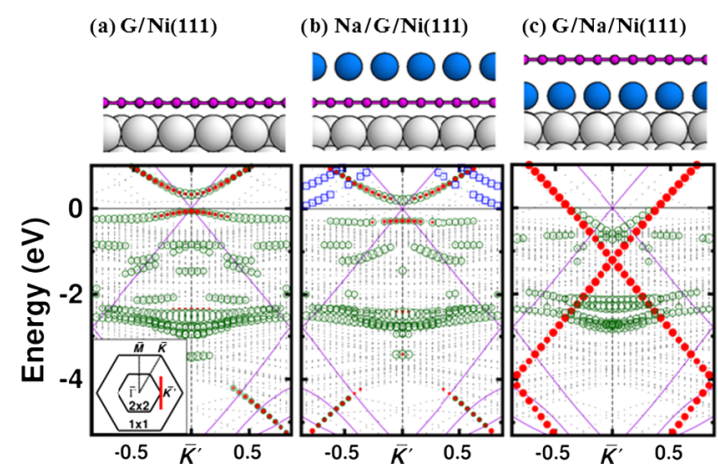

FIG. 4. Majority-spin band structures of (a) graphene/Ni(111), (b) $\mathrm{Na}(0.63 \mathrm{ML}) /$ graphene/Ni(111), and (c) graphene/ $\mathrm{Na}(0.63 \mathrm{ML}) / \mathrm{Ni}(111)$. The vertical solid line in the surface Brillouin zone represents the calculated line. The Fermi energy is set to 0 , and the bands of ideal graphene are given as the reference by the thin solid lines. The filled red (open green) circles represent $\mathrm{C}$-derived (Ni-derived) surface states that contain more than $20 \%(40 \%)$ of charge in the graphene layer (topmost $\mathrm{Ni}$ layer). The size of the circles is proportional to the amount of charge localized in the graphene and the Ni layer. Open blue squares represent the $\mathrm{Na}$-derived surface states that contain more than $5 \%$ of charge in the $\mathrm{Na}$ layer.

Dirac-cone shape of ideal graphene. The Dirac point shifts down with coverage up to $0.63 \mathrm{ML}$. The shift depends on the amount of charge transfer to the graphene layer, and it would be expected to be proportional with the Na coverage in the low-coverage regime. After 0.63 ML, the downward shift of the Dirac point is almost saturated at about $-1.2 \mathrm{eV}$, implying that additional Na doping little affects the graphene $\pi$ bands. For this reason, we will focus on the $\mathrm{Na}$ coverage of $0.63 \mathrm{ML}$ in the following band structure.

Figure 4 shows the majority-spin band structures of the graphene/Ni(111) surface before and after 0.63-ML Na deposition. In Fig. 4(a), the important feature of the graphene/Ni(111) band structure is that the Dirac-cone shape of graphene $\pi$ bands is significantly disturbed: The $\pi$ band dispersions are not linear any longer with a gap opening of about $0.4 \mathrm{eV}$ through a strong hybridization with the Ni $d$ states near the Fermi level. Interestingly, in Fig. 4(b), the adsorbed $\mathrm{Na}$ atoms little affect the electronic structure of graphene/ $\mathrm{Ni}(111)$. The adsorbed $\mathrm{Na}$ atoms form metallic bands (still mostly empty at this coverage), but there is no strong hybridization between the Na-derived states and the graphene $\pi$ bands. The graphene bands are slightly changed with a small shift of $-0.21 \mathrm{eV}(-0.12 \mathrm{eV})$ at the $K^{\prime}$ point in filled (empty) states and a small increase in the $\pi$ band gap of $0.5 \mathrm{eV}$. In contrast, as seen in Fig. 4(c), Na intercalation perfectly recovers the Dirac-cone shape of ideal graphene. We find that, due to the interaction with $\mathrm{Na}$, the graphene bands shift downward by approximately $1.2 \mathrm{eV}$ from the Fermi level, indicating a large charge transfer from the $\mathrm{Na} / \mathrm{Ni}(111)$ surface to the graphene layer. While the $\mathrm{Na}$ atoms interact strongly with the $\mathrm{Ni}$ substrate, the $\mathrm{Na}-\mathrm{Na}$ interaction is so weak that the Na-derived states are empty at approximately $3 \mathrm{eV}$ above the Fermi level.

\section{EXPERIMENTAL RESULTS AND DISCUSSION}

\section{A. Experimental details}

A clean $\mathrm{Ni}(111)$ surface is prepared by applying many successive cycles of $\mathrm{Ar}^{+}$sputtering for $30 \mathrm{~min}$ and then heating for $2 \mathrm{~min}$ at approximately $700^{\circ} \mathrm{C}$ in an ultrahighvacuum chamber. After confirming the $\mathrm{Ni}(111)$ surface cleanliness with low-energy electron diffraction and ARPES, a graphene layer is grown in an ambient $\mathrm{C}_{2} \mathrm{H}_{2}$ pressure of $1.0-5.0 \times 10^{-6}$ Torr and a sample temperature of approximately $700^{\circ} \mathrm{C}$. The ARPES studies are first performed at the beam line $10 \mathrm{D}$ and later reproduced at the beam line 4A2 of the Pohang Accelerator Laboratory (PAL), equipped with a Scienta R4000 electron analyzer, which provides an overall energy resolution of approximately $50 \mathrm{meV}$ at approximately $34 \mathrm{eV}$. All photoemission measurements are made at a pressure of $1.0 \times 10^{-10}$ Torr. The temperature-dependent ARPES and core-level PES data are taken using the PHOIBOS 150 analyzer at the 10D beam line of PAL. We use a 3-mm entrance slit of the analyzer to monitor the change in band structure within a very short time upon transition from adsorption to intercalation. So, the band gap looks exaggerated in the ARPES data. The sample is cooled down to $-140^{\circ} \mathrm{C}$ by liquid nitrogen. A commercial (SAES) getter source is used to deposit Na atoms on the graphene. The STM experiments are performed at room temperature. In order to make a clean Ni surface and graphene, the same method employed in the ARPES experiments is used.

\section{B. ARPES}

The graphene on the Ni surface intercalated with $\mathrm{Na}$ atoms has theoretically no band gap, whereas a large energy gap of approximately $1.3 \mathrm{eV}$ was observed experimentally [27]. This discrepancy might be due not only to the lower energy and angle resolutions but also to the sample that is still interacting with the substrate. So, we have revisited ARPES experiments. We have performed more detailed ARPES experiments, including coverage and temperature dependency, as well as core-level PES and STM experiments.

Figure 5 shows the electronic band structure measured by ARPES of the as-grown graphene on $\mathrm{Ni}(111)$ and the graphene/Ni(111) intercalated with $\mathrm{Na}$ near the $\Gamma$ and $K$ points. By inspecting the energy band dispersions of the as-grown graphene/Ni(111) sample shown in Figs. 5(a) and 5(c), the binding energies (BEs) of the $\pi$ state are observed to be about -10 and $-2.7 \mathrm{eV}$ at the $\Gamma$ and $K$ points, respectively, in agreement with previously reported values $[18,40]$. This can be explained by strong hybridization of the $\pi$ orbitals of graphene and the $d$ orbitals of the 

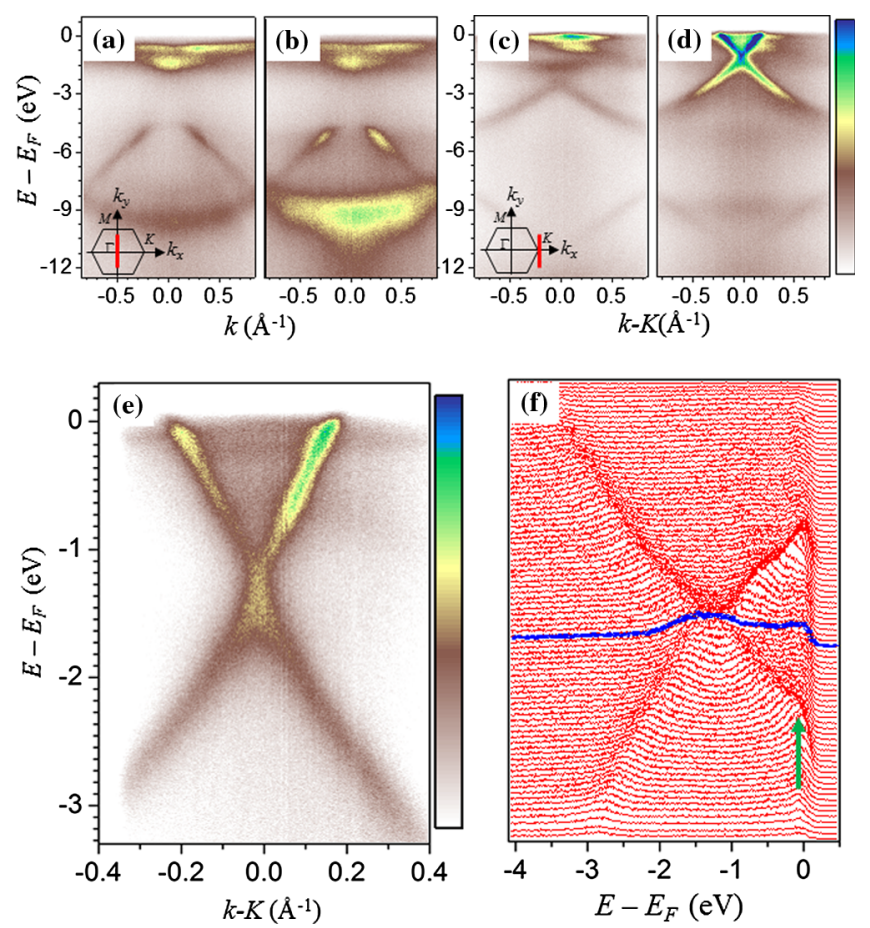

FIG. 5. Electronic band structures measured by ARPES of the (a),(c) as-grown graphene and (b),(d) Na-intercalated graphene, and (e),(f) high angular and energy-resolution measurements of the Na-intercalated graphene. (a),(b) Taken at the $\Gamma$ point; (c),(d) taken at the $K$ point. The data are measured along the red lines, as indicated in the insets. The color-scale bars in (a) and (b) are the same. (e) Band structure around the $K$ point near the Fermi energy, taken along the direction perpendicular to the $\Gamma-K$. (f) Photoemission intensity profiles as a function of BE (energydistribution curves).

$\mathrm{Ni}(111)$ metal surface. The $3 d$ states of the clean $\mathrm{Ni}$ metal are also shown at BEs of about -0.2 and $-1.6 \mathrm{eV}$.

Figures 5(b) and 5(d) show the electronic band structures after Na intercalation near the $\Gamma$ and $K$ points, respectively. While the $\sigma, \pi$, and $\mathrm{Ni} 3 d$ bands are not considerably changed near the $\Gamma$ point, a characteristic $\pi$ band appears near the $K$ point. The introduction of $\mathrm{Na}$ atoms to the interface between the graphene and the $\mathrm{Ni}(111)$ surface weakens the strong hybridization between $\mathrm{C} 2 p$ and $\mathrm{Ni} 3 d$ orbitals, causing the increase of distance between the graphene and the metal substrate. Because the graphene layer detaches from the $\mathrm{Ni}(111)$ surface, the linear band dispersion is completely recovered.

Figure 5(e) shows a close-up of the energy band structure of Na-deposited graphene/Ni(111) near the Fermi energy, as measured by ARPES with higher energy and momentum resolutions. It clearly shows two single $\pi$ bands that meet at the Dirac point located at about $-1.25 \mathrm{eV}$, indicating a negligible band-gap opening. Even if the amount of Na adsorption is changed, the resulting electronic band structures do not significantly change; two bands of as-grown and Na-deposited graphene at low coverage are simultaneously observed. This will be discussed more later. The $\mathrm{Na}$ atoms break the chemical interaction of the graphene and the Ni substrate, changing the electronic structure of the graphene/ $\mathrm{Ni}(111)$ system toward that of a quasi-free-standing graphene. According to our DFT calculations, a simple adsorption of $\mathrm{Na}$ atoms on top of the graphene/Ni(111) system does not recover the Dirac-cone band structure of graphene. As will be shown below, the adsorbed $\mathrm{Na}$ atoms fully penetrate beneath the graphene layer.

Individual energy-distribution curves of the Na-intercalated graphene/Ni(111) are shown in Fig. 5(f) with the $K^{\prime}$-point spectrum indicated in blue. No distinct separation between the valence and conduction bands near the Dirac point is observed. In addition, the energy-distribution curves indicate that kinks appear around the $K^{\prime}$ point. These kinks originate from a finite self-energy due to many-body interactions. This observation agrees well with previous reports in two ways [41]. First, a strong kink [denoted by the arrow in Fig. 5(f)] is observed at a BE of $0.2 \mathrm{eV}$ below the Fermi energy, which is due to renormalization of the electron bands by electron-phonon coupling [41]. Second, the actual linear band deviates from the ideal dispersion (or singleparticle prediction) near the Dirac energy. This deviation can originate from electron-plasmon scattering due to manybody effects. Similar behaviors were reported theoretically from first-principles calculations [35] and also in experimental results of graphene with chemical dopants [42]. However, the small band-gap opening could not be entirely neglected [43].

Figures 6(a) and 6(b) show the electronic structure of the graphene grown on the $\mathrm{Ni}(111)$ surface at the $\Gamma$ and $K$ points, respectively, after depositing a small amount of $\mathrm{Na}$ atoms at room temperature. While the structure at the $\Gamma$ point is almost the same, that at the $K$ point is considerably changed. The original $\pi$ band becomes faint, and two additional bands that meet at a Dirac point appear. This

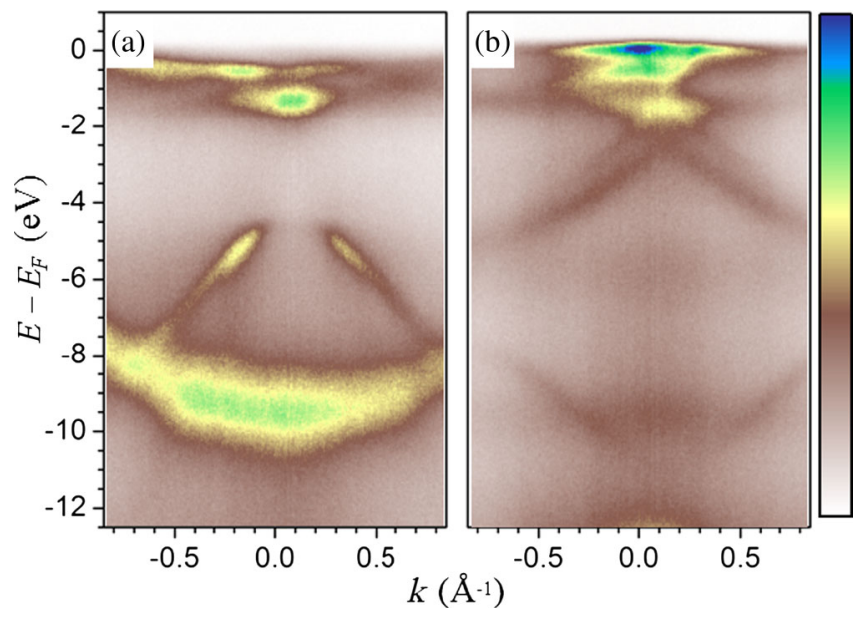

FIG. 6. Electronic band structures of the graphene with a low coverage of $\mathrm{Na}$ atoms taken at (a) the $\Gamma$ and (b) the $K$ points. 

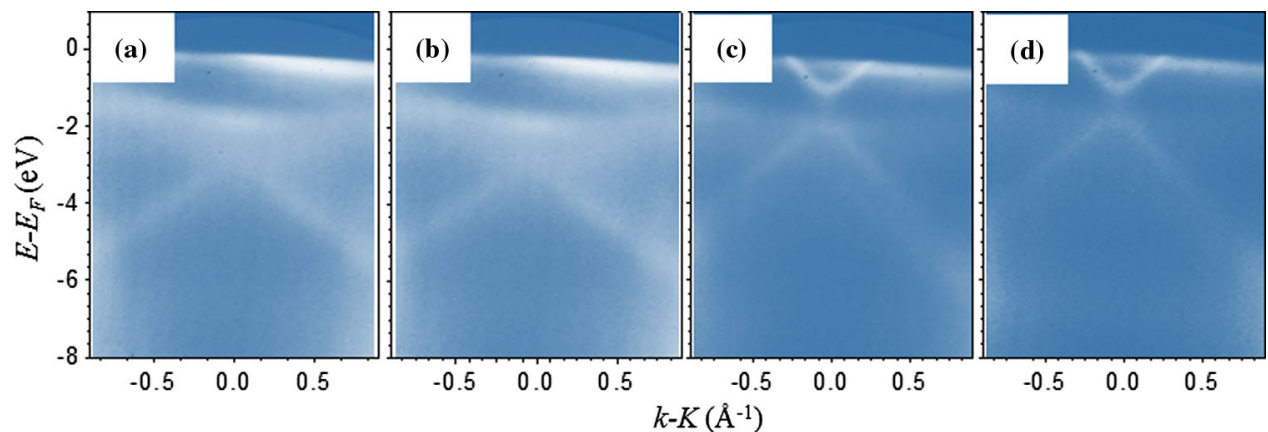

FIG. 7. Temperature-dependent ARPES data of the graphene measured at the $K$ point (a) at $-140^{\circ} \mathrm{C}$, (b) after Na deposition at $-140{ }^{\circ} \mathrm{C}$, (c) with the temperature increased to $-100^{\circ} \mathrm{C}$ after the $\mathrm{Na}$ deposition, and (d) with the temperature increased to $+20^{\circ} \mathrm{C}$ after the $\mathrm{Na}$ deposition.

indicates that a small amount of $\mathrm{Na}$ atoms is intercalated and recovers the Dirac-cone shape at local regions.

This result seems to be closely related with a tiny diffusion barrier for $\mathrm{Na}$ atoms. As explained in the theoretical part, the diffusion barrier is as small as 0.03 $\mathrm{eV}$ for $\mathrm{Na}$ atoms on the graphene/ $\mathrm{Ni}(111)$ surface. Therefore, $\mathrm{Na}$ atoms spontaneously intercalate and then form a stable structure as stable as that at the Na saturation coverage locally, leading to two different band structures from both Na-intercalated and clean graphene regions. One might expect an overlayer adsorption on graphene at a lower temperature than room temperature by restricting surface diffusion. We have accordingly performed temperature-dependent ARPES and core-level PES experiments.

\section{Temperature-dependent ARPES and core-level PES}

Figure 7 shows temperature-dependent ARPES data taken using the PHOIBOS 150 analyzer at the 10D beam line of the PAL. After confirming the formation of graphene by measuring the energy band at the $\Gamma$ and $K$ points, the sample is cooled down to $-140{ }^{\circ} \mathrm{C}$ by liquid nitrogen. Figure 7(a) shows the band structure around the $K$ point at $-140^{\circ} \mathrm{C}$. Ni $3 d$ and the $\pi$ band of graphene are observed. Although $\mathrm{Na}$ atoms are deposited on the graphene layer at $-140^{\circ} \mathrm{C}$, the band structure near the $K$ point does not change, as shown in Fig. 7(b). When the temperature reaches around $-100{ }^{\circ} \mathrm{C}$, however, the band structure is considerably changed; that is, the linear bands appear as shown in Fig. 7(c), indicating that the $\mathrm{Na}$ atoms are intercalated between the graphene and the $\mathrm{Ni}$ surface. No change in the band structure is observed with increasing temperature up to $+20^{\circ} \mathrm{C}$, as shown in Fig. 7(d).

The temperature dependence of the Na intercalation is also confirmed by PES spectra for $\mathrm{C} 1 s$ and $\mathrm{Na} 2 p$ core levels. Figure 8 shows PES spectra taken with a photon energy of $360 \mathrm{eV}$ from the clean graphene grown on the $\mathrm{Ni}$ surface measured at $-140^{\circ} \mathrm{C}$ (solid black line). The Ni $3 p$ and $\mathrm{Ni} 4 d$ peaks are observed near 66 and $0 \mathrm{eV}$, respectively. A sharp C $1 s$ peak is found near approximately $284.5 \mathrm{eV}$ due to the presence of a single-layer graphene.
The intensity of the $\mathrm{C} 1 s$ peak is reduced, and a $\mathrm{Na} 2 p$ peak appears at approximately $31 \mathrm{eV}$ after $\mathrm{Na}$ deposition at $-140{ }^{\circ} \mathrm{C}$ (solid red line), indicating that the graphene is covered with $\mathrm{Na}$ atoms. The intensity of the $\mathrm{C} 1 s$ peak increases, while that of the $\mathrm{Na} 2 p$ peak decreases with increasing temperature up to $+20^{\circ} \mathrm{C}$ (solid green line). The C $1 s / \mathrm{Na} 2 p$ peak intensity ratio changes from 3.72 at $-140{ }^{\circ} \mathrm{C}$ to 4.23 at $+20^{\circ} \mathrm{C}$. This means that the $\mathrm{Na}$ atoms adsorbed on the graphene at $-140^{\circ} \mathrm{C}$ penetrate below the layer as temperature increases above the critical temperature.

\section{STM}

It is characterized by STM to find out whether $\mathrm{Na}$ atoms are intercalated between the graphene and the substrate or

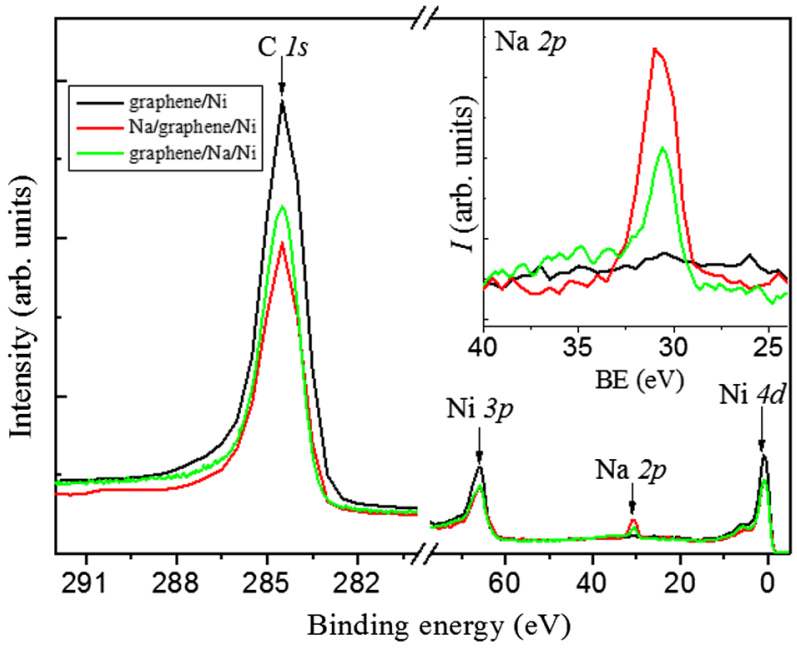

FIG. 8. Core-level PES spectra of the graphene grown on the $\mathrm{Ni}$ surface taken with a photon energy of $360 \mathrm{eV}$. The black, red, and green lines represent the PES spectra of the graphene/Ni(111) at $-140{ }^{\circ} \mathrm{C}, \mathrm{Na} /$ graphene/ $\mathrm{Ni}(111)$ at $-140{ }^{\circ} \mathrm{C}$, and graphene $/ \mathrm{Na} /$ $\mathrm{Ni}(111)$ at $+20^{\circ} \mathrm{C}$, respectively. The intensity ratio $\mathrm{C} 1 s / \mathrm{Na} 2 p$ is 3.72 for the $\mathrm{Na} / \mathrm{graphene} / \mathrm{Ni}(111)$ at $-140^{\circ} \mathrm{C}$ and 4.23 for the graphene/ $\mathrm{Na} / \mathrm{Ni}(111)$ at $+20^{\circ} \mathrm{C}$, respectively. The inset depicts the Na $2 p$ PES spectra of the surface. 

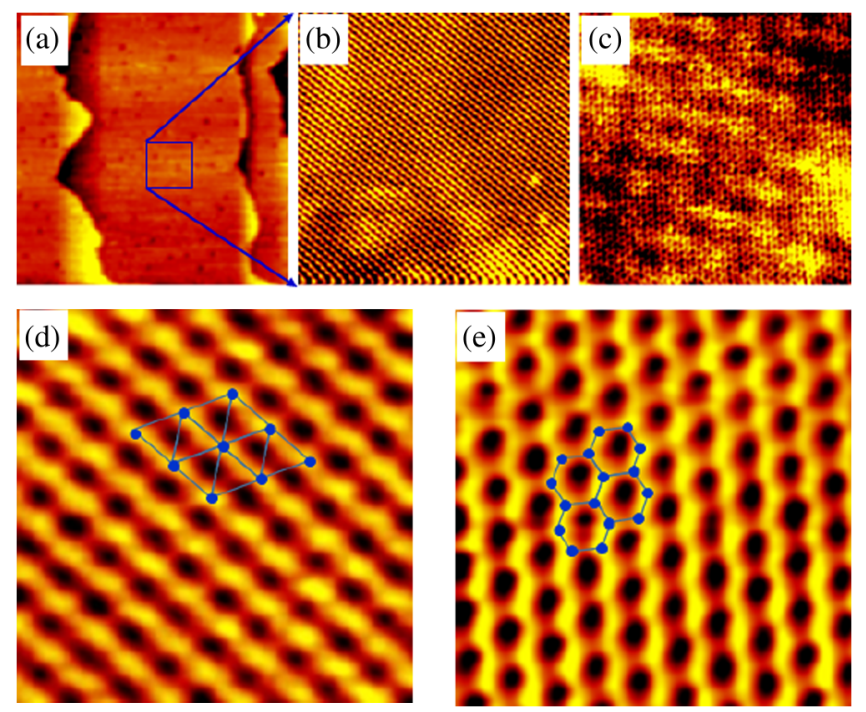

FIG. 9. STM topography images of a graphene grown on the Ni surface. (a) Large-area scan of the graphene before $\mathrm{Na}$ intercalation: $2000 \times 2000 \AA, I_{t}=0.6 \mathrm{nA}$, and $V_{t}=26.9 \mathrm{mV}$. (b) Zoomed-in STM image of the squared region in (a): $100 \times 100 \AA, I_{t}=2.59 \mathrm{nA}$, and $V_{t}=100 \mathrm{mV}$. (c) STM image after $\mathrm{Na}$ intercalation: $100 \times 100 \AA, \quad I_{t}=3.88 \mathrm{nA}$, and $V_{t}=41.5 \mathrm{mV}$. (d) An atomically resolved STM image of (b): $20 \times 20 \AA, I_{t}=2.59 \mathrm{nA}$, and $V_{t}=42.4 \mathrm{mV}$. (e) An atomically resolved STM image of (c): $30 \times 30 \AA, I_{t}=3.20 \mathrm{nA}$, and $V_{t}=41.5 \mathrm{mV}$.

adsorbed on the graphene. Figure 9(a) shows the STM image of a large area of graphene on the Ni surface taken at room temperature. No domain boundary is visible on the terrace. In contrast, on the $\mathrm{Cu}$ surface, the domain size is quite small with many domain boundaries [44]. Figure 9(b) shows a zoomed-in STM image of the squared region in Fig. 9(a), where the graphene image is clearly observed. An atomically well-resolved STM image of the graphene on the Ni surface is shown in Fig. 9(d). The threefold symmetry is clearly observed due to the two different carbon adsorption sites, the top and fcc sites of Ni. Figure 9(c) shows the STM image of the graphene after Na intercalation, where a superstructure is observed. Figure 9(e) shows the atomically resolved STM image of the graphene after $\mathrm{Na}$ deposition at room temperature, which provides further evidence of the $\mathrm{Na}$ intercalation between the $\mathrm{Ni}$ surface and the graphene. The Na-induced decoupling of the graphene from the substrate changes the threefold symmetry into the sixfold symmetry, which freestanding graphene should have.

It has been reported that a monolayer graphene membrane is impermeable to standard gases, including helium [45]. Nevertheless, this work demonstrates that the intercalation of some alkali metals between graphene and metal substrates is achievable, possibly through domain boundaries and point defects of the graphenes that may play a key role in such metal-atom intercalations into the interface. In particular, because alkali metals can be easily oxidized, the intercalation layer can be made insulating and thus separate the graphene farther from the substrate. Therefore, we do not need the process to transfer graphene to other substrates to recover the inherent electronic structure of graphene by removing metals with acids (which has often resulted in undesirable tears, defects, and impurities in graphene).

\section{CONCLUSION}

In conclusion, we report a spontaneous $\mathrm{Na}$ intercalation, recovering the electronic structure of graphene from a strongly interacting $\mathrm{Ni}$ substrate that is characterized by using DFT calculations and ARPES, PES, and STM measurements. The introduction of $\mathrm{Na}$ atoms on the graphene/ $\mathrm{Ni}(111)$ surface energetically prefers intercalation into the interface over simple surface adsorption. Unlike most intercalants, such as $\mathrm{Au}, \mathrm{Al}$, etc., $\mathrm{Na}$ atoms adsorbed on the graphene surface penetrate into the graphene far below room temperature (approximately $-100^{\circ} \mathrm{C}$ ) and thus the intercalation takes place spontaneously even at room temperature. This seems to be related closely with the tiny diffusion barrier for $\mathrm{Na}$ atoms. The spontaneous intercalation makes the graphene layer nearly electronically ideal, thereby showing its inherent sixfold symmetry and characteristic bands featuring the Dirac-cone structure with a negligible band gap. The intercalated $\mathrm{Na}$ atoms could be easily oxidized to become an insulator. Thus, it provides new possibilities toward the high-quality, large-area, and quasi-free-standing graphene on metal or insulating substrates without etching and transferring processes.

\section{ACKNOWLEDGMENTS}

This work was supported by the National Research Foundation of Korea (NRF) through the SRC Center for Topological Matter (No. 2011-0030787) and the National Honor Scientist Program (2010-0020414), and by KISTI (KSC-2011-G3-02) and the Basic Science Research Program (No. 2011-0008907). C. C. H. also acknowledges financial support by the NRF (No. 2009-0083380 and No. 2011-0009427) and by projects of POSCO. The experiments at PLS were supported in part by MSIP and POSTECH.

[1] K. S. Novoselov, A. K. Geim, S. V. Morozov, D. Jiang, Y. Zhang, S. V. Dubonos, I. V. Grigorieva, and A. A. Firsov, Electric Field Effect in Atomically Thin Carbon Films, Science 306, 666 (2004).

[2] H.-G. Jee, K.-H. Jin, J.-H. Han, H.-N. Hwang, S.-H. Jhi, Y. D. Kim, and C.-C. Hwang, Controlling the Self-Doping of Epitaxial Graphene on SiC via Ar Ion Treatment, Phys. Rev. B 84, 075457 (2011).

[3] C. Berger, Z. Song, X. Li, X. Wu, N. Brown, C. Naud, D. Mayou, T. Li, J. Hass, A. N. Marchenkov, E. H. Conrad, 
P. N. First, and Walt A. de. Heer, Electronic Confinement and Coherence in Patterned Epitaxial Graphene, Science 312, 1191 (2006).

[4] K. V. Emtsev, A. Bostwick, K. Horn, J. Jobst, G. L. Kellogg, L. Ley, J. L. McChesney, T. Ohta, S. A. Reshanov, J. Röhrl, E. Rotenberg, A. K. Schmid, D. Waldmann, H. B. Weber, and T. Seyller, Towards Wafer-Size Graphene Layers by Atmospheric Pressure Graphitization of Silicon Carbide, Nat. Mater. 8, 203 (2009).

[5] P. W. Sutter, J.-I. Flege, and E. A. Sutter, Epitaxial Graphene on Ruthenium, Nat. Mater. 7, 406 (2008).

[6] A. Reina, X. Jia, J. Ho, D. Nezich, H. Son, V. Bulovic, M. S. Dresselhaus, and J. Kong, Large Area, Few-Layer Graphene Films on Arbitrary Substrates by Chemical Vapor Deposition, Nano Lett. 9, 30 (2009).

[7] K. S. Kim, Y. Zhao, H. Jang, S. Y. Lee, J. M. Kim, K. S. Kim, J.-H. Ahn, P. Kim, J.-Y. Choi, and B. H. Hong, LargeScale Pattern Growth of Graphene Films for Stretchable Transparent Electrodes, Nature (London) 457, 706 (2009).

[8] X. Li, W. Cai, J. An, S. Kim, J. Nah, D. Yang, R. Piner, A. Velamakanni, I. Jung, E. Tutuc, S. K. Banerjee, L. Colombo, and R. S. Ruoff, Large-Area Synthesis of High-Quality and Uniform Graphene Films on Copper Foils, Science 324, 1312 (2009).

[9] J. Cho, L. Gao, J. Tian, H. Cao, W. Wu, Q. Yu, E. N. Yitamben, B. Fisher, J. R. Guest, Y. P. Chen, and N. P. Guisinger, Atomic-Scale Investigation of Graphene Grown on $\mathrm{Cu}$ Foil and the Effects of Thermal Annealing, ACS Nano 5, 3607 (2011).

[10] X. Li, W. Cai, L. Colombo, and R. S. Ruoff, Evolution of Graphene Growth on $\mathrm{Ni}$ and $\mathrm{Cu}$ by Carbon Isotope Labeling, Nano Lett. 9, 4268 (2009).

[11] Y. Lee, S. Bae, H. Jang, S. Jang, S.-E. Zhu, S. H. Sim, Y. I. Song, B. H. Hong, and J.-H. Ahn, Wafer-Scale Synthesis and Transfer of Graphene Films, Nano Lett. 10, 490 (2010).

[12] S. Bae, H. Kim, Y. Lee, X. Xu, J.-S. Park, Y. Zheng, J. Balakrishnan, T. Lei, H. R. Kim, Y. I. Song, Y.-J. Kim, K. S. Kim, B. Ozyilmaz, J.-H. Ahn, B. H. Hong, and S. Iijima, Roll-to-Roll Production of 30-Inch Graphene Films for Transparent Electrodes, Nat. Nanotechnol. 5, 574 (2010).

[13] P. Y. Huang, C. S. Ruiz-Vargas, A. M. van der Zande, W. S. Whitney, M. P. Levendorf, J. W. Kevek, S. Garg, J. S. Alden, C. J. Hustedt, J. Zhu, J. Park, P. L. McEuen, and D. A. Muller, Grains and Grain Boundaries in Single-Layer Graphene Atomic Patchwork Quilts, Nature (London) 469, 389 (2011).

[14] C. Jeon, H.-N. Hwang, W. G. Lee, Y. G. Jung, K. S. Kim, C.-Y. Park, and C.-C. Hwang, Rotated Domains in Chemical Vapor Deposition-Grown Monolayer Graphene on $\mathrm{Cu}$ (111): and Angle-Resolved Photoemission Study, Nanoscale 5, 8210 (2013).

[15] J. Lahiri, Y. Lin, P. Bozkurt, I. I. Oleynik, and M. Batzill, An Extended Defect in Graphene as a Metallic Wire, Nat. Nanotechnol. 5, 326 (2010).

[16] Y. Cho, Y. C. Choi, and K. S. Kim, Graphene Spin-Valve Device Grown Epitaxially on the Ni(111) Substrate: A First Principles Study, J. Phys. Chem. C 115, 6019 (2011).

[17] Z. H. Ni, L. A. Ponomarenko, R. R. Nair, R. Yang, S. Anissimova, I. V. Grigorieva, F. Schedin, P. Blake, Z. X. Shen, E. H. Hill, K. S. Novoselov, and A. K. Geim, On
Resonant Scatterers as a Factor Limiting Carrier Mobility in Graphene, Nano Lett. 10, 3868 (2010).

[18] W. H. Lee, J. Park, S. H. Sim, S. Lim, K. S. Kim, B. H. Hong, and K. Cho, Surface-Directed Molecular Assembly of Pentacene on Monolayer Graphene for High-Performance Organic Transistors, J. Am. Chem. Soc. 133, 4447 (2011).

[19] A. M. Shikin, G. V. Prudnikova, V. K. Adamchuk, F. Moresco, and K.-H. Rieder, Surface Intercalation of Gold underneath a Graphite Monolayer on Ni(111) Studied by Angle-Resolved Photoemission and High-Resolution Electron-Energy-Loss Spectroscopy, Phys. Rev. B 62, 13202 (2000).

[20] Yu. S. Dedkov, A. M. Shikin, V. K. Adamchuk, S. L. Molodtsov C. Laubschat, A. Bauer, and G. Kaindl, Intercalation of Copper underneath a Monolayer of Graphite on Ni(111), Phys. Rev. B 64, 035405 (2001).

[21] A. Varykhalov, J. Sánchez-Barriga, A. M. Shikin, C. Biswas, E. Vescovo, A. Rybkin, D. Marchenko, and O. Rader, Electronic and Magnetic Properties of Quasifreestanding Graphene on Ni, Phys. Rev. Lett. 101, 157601 (2008).

[22] A. G. Starodubov, M. A. Medvetski, A. M. Shikin, and V. K. Adamchuk, Intercalation of Silver Atoms under a Graphite Monolayer on Ni(111), Phys. Solid State 46, 1340 (2004).

[23] A. M. Shikin, V. K. Adamchuk, and K. H. Rieder, Formation of Quasi free Graphene on the Ni(111) Surface with Intercalated $\mathrm{Cu}, \mathrm{Ag}$, and $\mathrm{Au}$ Layers, Phys. Solid State 51, 2390 (2009).

[24] A. Varykhalov, M. R. Scholz, T. K. Kim, and O. Rader, Effect of Noble-Metal Contacts on Doping and Band Gap of Graphene, Phys. Rev. B 82, 121101 (2010).

[25] M. H. Kang, S. C. Jung, and J. W. Park, Density Functional Study of the Au-Intercalated Graphene/Ni(111) Surface, Phys. Rev. B 82, 085409 (2010).

[26] E. N. Voloshina, A. Generalov, M. Weser, S. Bottcher, K. Horn, and Yu. S. Dedkov, Structural and Electronic Properties of the Graphene/Al/Ni(111) Intercalation System, New J. Phys. 13, 113028 (2011).

[27] A. Nagashima, N. Tejima, and C. Oshima, Electronic States of the Pristine and Alkali-Metal-Intercalated Monolayer Graphite/Ni(111) Systems, Phys. Rev. B 50, 17487 (1994).

[28] A. Grüneis and D. V. Vyalikh, Tunable Hybridization between Electronic States of Graphene and a Metal Surface, Phys. Rev. B 77, 193401 (2008).

[29] G. Kresse and J. Furthmuller, Efficient Iterative Schemes for Ab Initio Total-Energy Calculations Using a Plane-Wave Basis Set, Phys. Rev. B 54, 11169 (1996).

[30] P. E. Blöchl, Projector Augmented-Wave Method, Phys. Rev. B 50, 17953 (1994).

[31] G. Kresse and D. Joubert, From Ultrasoft Pseudo potentials to the Projector Augmented-Wave Method, Phys. Rev. B 59, 1758 (1999).

[32] Y. Gamo, A. Nagashima, M. Wakabayashi, M. Terai, and C. Oshima, Atomic Structure of Monolayer Graphite Formed on Ni(111), Surf. Sci. 374, 61 (1997).

[33] G. Karpan, L. Calmels, A. Altibelli, and V. Serin, FirstPrinciples Calculation of the Electronic Structure and EELS Spectra at the Graphene/Ni(111) Interface, Phys. Rev. B 71, 075402 (2005). 
[34] V. M. Karpan, G. Giovannetti, P. A. Khomyakov, M. Talanana, A. A. Starikov, M. Zwierzycki, J. van den Brink, G. Brocks, and P. J. Kelly, Graphite and Graphene as Perfect Spin Filters, Phys. Rev. Lett. 99, 176602 (2007).

[35] M. Fuentes-Cabrera, M. I. Baskes, A. V. Melechko, and M. L. Simpson, Bridge Structure for the Graphene/Ni(111) System: A First Principles Study, Phys. Rev. B 77, 035405 (2008).

[36] R. Balog, B. Jørgensen, L. L. Nilsson, M. Andersen, E. Rienks, M. Bianchi, M. Fanetti, E. Lægsgaard, A. Baraldi, S. Lizzit, Z. Sljivancanin, F. Besenbacher, B. Hammer, T. G. Pedersen, P. Hofmann, and L. Hornekær, Bandgap Opening in Graphene Induced by Patterned Hydrogen Adsorption, Nat. Mater. 9, 315 (2010).

[37] D. Haberer, D. V. Vyalikh, S. Taioli, B. Dora, M. Farjam, J. Fink, D. Marchenko, T. Pichler, K. Ziegler, S. Simonucci, M. S. Dresselhaus, M. Knupfer, B. Búchner, and A. Grüneis, Tunable Band Gap in Hydrogenated Quasifree-standing Graphene, Nano Lett. 10, 3360 (2010).

[38] C. Riedl, C. Coletti, T. Iwasaki, A. A. Zakharov, and U. Starke, Quasi-free-standing Epitaxial Graphene on SiC Obtained by Hydrogen Intercalation, Phys. Rev. Lett. 103, 246804 (2009).

[39] S. Watcharinyanon, L. I. Johansson, C. Xia, and C. Virojanadara, Changes in Structural and Electronic
Properties of Graphene Grown on 6H-SiC(0001) Induced by Na Deposition, J. Appl. Phys. 111, 083711 (2012).

[40] V. Varykhalov, D. Marchenko, J. Sanchez-Barriga, M. R. Scholz, B. Verberck, B. Trauzettel, T. O. Wehling, C. Carbone, and R. Rader, Intact Dirac Cones at Broken Sublattice Symmetry: Photoemission Study of Graphene on Ni and Co, Phys. Rev. X 2, 041017 (2012).

[41] A. Bostwick, T. Ohta, T. Seyller, K. Horn, and E. Rotenberg, Quasiparticle Dynamics in Graphene, Nat. Phys. 3, 36 (2007).

[42] C.-H. Park, F. Giustino, C. D. Spataru, M. L. Cohen, and S. G. Louie, Charge Sensing and Controllable Tunnel Coupling in a Si/SiGe Double Quantum Dot, Nano Lett. 9, 4234 (2009).

[43] S. Y. Zhou, G.-H. Gweon, A. V. Fedorov, P. N. First, W. A. de Heer, D.-H. Lee, F. Guinea, A. H. Castroneto, and A. Lanzara, Substrate-Induced Bandgap Opening in Epitaxial Graphene, Nat. Mater. 6, 770 (2007).

[44] S. U. Yu, Y. Cho, B. Park, N. Kim, I. S. Youn, M. Son, J. K. Kim, H. C. Choi, and K. S. Kim, Fast Benchtop Visualization of Graphene Grain Boundaries Using Adhesive Properties of Defects, Chem. Commun. (Cambridge) 49, 5474 (2013).

[45] J. S. Bunch, S. S. Verbridge, J. S. Alden, A. M. van der Zande, J. M. Parpia, H. G. Craighead, and P. L. McEuen, Impermeable Atomic Membranes from Graphene Sheets, Nano Lett. 8, 2458 (2008). 